\title{
Numerical Solution of a Moving Boundary Problem of One-Dimensional Flow in Semi-Infinite Long Porous Media with Threshold Pressure Gradient
}

\author{
Jun Yao, ${ }^{1}$ Wenchao Liu, ${ }^{1}$ and Zhangxin Chen ${ }^{2}$ \\ ${ }^{1}$ School of Petroleum Engineering, China University of Petroleum (East China), Qingdao 266580, China \\ ${ }^{2}$ Department of Chemical and Petroleum Engineering, Schulich School of Engineering,University of Calgary, \\ Calgary, AB, Canada T2N 1N4 \\ Correspondence should be addressed to Jun Yao; rcogfr_upc@126.com
}

Received 15 July 2013; Revised 28 September 2013; Accepted 18 October 2013

Academic Editor: Kue-Hong Chen

Copyright (C) 2013 Jun Yao et al. This is an open access article distributed under the Creative Commons Attribution License, which permits unrestricted use, distribution, and reproduction in any medium, provided the original work is properly cited.

\begin{abstract}
A numerical method is presented for the solution of a moving boundary problem of one-dimensional flow in semi-infinite long porous media with threshold pressure gradient (TPG) for the case of a constant flow rate at the inner boundary. In order to overcome the difficulty in the space discretization of the transient flow region with a moving boundary in the process of numerical solution, the system of partial differential equations for the moving boundary problem is first transformed equivalently into a closed system of partial differential equations with fixed boundary conditions by a spatial coordinate transformation method. Then a stable, fully implicit finite difference method is adopted to obtain its numerical solution. Finally, numerical results of transient distance of the moving boundary, transient production pressure of wellbore, and formation pressure distribution are compared graphically with those from a published exact analytical solution under different values of dimensionless TPG as calculated from actual experimental data. Comparison analysis shows that numerical solutions are in good agreement with the exact analytical solutions, and there is a big difference of model solutions between Darcy's flow and the fluid flow in porous media with TPG, especially for the case of a large dimensionless TPG.
\end{abstract}

\section{Introduction}

With the increase of international oil price, unconventional reservoirs such as low-permeable reservoirs, heavy oil reservoirs, and shale gas reservoirs [1] have become new development targets in the field of petroleum engineering in modern times. The relevant research on the kinematic principles of the fluid flow in unconventional reservoirs is a recently hot topic. Abundant experimental and theoretical analyses [2-15] have demonstrated that the fluid flow in low-permeable reservoirs and the Bingham non-Newtonian fluid flow in heavy oil reservoirs do not obey the conventional Darcy's law; there exists a threshold pressure gradient (TPG) or the yield stress. That is, the fluid flow happens only if the formation pressure gradient exceeds the TPG or the shear stress for the fluid is larger than the yield stress.
Many literatures have demonstrated the significance of taking into account the TPG for the fluid flow in unconventional reservoirs. For example, Zhu et al. [16] conducted experimental investigation of gas flow in water-bearing tight gas reservoirs with TPG and analytical investigation of mathematical model of low-velocity gas flow; their calculation results indicate that the peripheral reserves of the wellbore are difficult to deploy, and the reservoir energy is mainly consumed near the wellbore due to existence of TPG, which is really unlike Darcy's flow. Zhu et al. [17] presented a method for improving history-matching precision of low-permeable reservoir numerical simulation by considering TPG, and relevant applications in Units X10 and X11 of Daqing Oilfield verify the obviously improved history-matching precision of reservoir numerical simulation. Yin and $\mathrm{Pu}$ [18] performed study on the surfactant flooding simulation for lowpermeable oilfield in the condition of TPG; the enhanced 
matching degree between theoretical model and field practice was verified through a pilot test of surfactant flooding in Chao 45 Block of Daqing Oilfield.

Due to the derivative discontinuity for the modified Darcy's law [2], the mathematical model for this physics, which the oilfield-development technologies such as well testing and reservoir numerical simulation for unconventional reservoirs involve, should be built as a moving boundary problem. Some analytical or numerical investigations [19-30] have demonstrated the big difference between the mathematical models with considering moving boundary conditions and those without considering moving boundary conditions. First of all, pressure distribution curves calculated from the mathematical model with moving boundary conditions due to the TPG show compact support $[29,30]$, which is similar to the characteristics of power-law nonNewtonian fluid flow $[20,22,25]$ in porous media or the fluid flow in nanoporous media [1]. Wu et al. [20] incorporated moving boundary conditions for theoretical study of the radial flow and displacement of a Bingham fluid in porous media with TPG by integral method and also presented a confirmed reservoir well-test-analysis method. Feng et al. [24] studied the model of unsteady radial flow in lowpermeable gas reservoirs considering the TPG by using Green function method with numerical approximation; the moving boundary can represent the single-well control radius; by using the data from Sichuan Gas Field, computation results show that the mathematical model with moving boundary conditions could correctly reflect the seepage mechanics and production performance for low-permeable gas reservoirs. Wang et al. [27, 28] studied the effect of moving boundary on the transient radial flow in low-permeable reservoirs with TPG and also demonstrated the significance of taking into account the effect of the moving boundary due to the TPG for engineering applications.

The moving boundary problem of fluid flow in porous media with TPG is different from the classical Stefan problem in heat conduction theory [29]: the velocity of the moving boundary is proportional to the second derivative of the unknown pressure function with respect to the distance at this moving boundary. However, due to the strong nonlinearity of the moving boundary problem, it is very difficult to obtain its exact analytical solution, whereas, recently, in the new published paper [29], exact analytical solutions for the non-Stefan moving boundary problems of one-dimensional flow in semi-infinite long porous media with TPG are presented through a similarity transformation method. Before the occurrence of the exact analytical solutions, approximate analytical solutions [19-21, 26] and numerical solutions [22$25,27,28,30]$ have been the main research tools for solving the non-Stefan moving boundary problems of the fluid flow in porous media with TPG. However, these solutions generally lack a strict verification with the related exact analytical solutions. Furthermore, some problems of fluid flow in porous media with threshold pressure are much more complicated and nonlinear such as the consideration of nonlinear terms in the governing equations [31] and the coupling of stress sensitive effect in low-permeable porous media [32], and then it will be extremely hard to obtain the exact analytical solutions. Therefore, it becomes very necessary to develop a verified numerical method by the published exact analytical solution [29] in order to solve more complicated moving boundary problems of fluid flow in porous media with TPG.

The objective of this paper is to present a simple and novel method for numerical solution of the moving boundary problem of one-dimensional flow in semi-infinite long porous media with TPG for the case of a constant flow rate at the inner boundary. First, this moving boundary problem is equivalently transformed into a closed partial differential equation system with fixed boundary conditions by the spatial coordinate transformation method [33-35]. Then a stable, fully implicit finite difference method [36] is adopted to obtain its numerical solution. Finally, the accuracy of the numerical solution is verified through graphically comparing with the published exact analytical solution. The numerical method presented here is applicable to the moving boundary problems of multidimensional flow in porous media with TPG.

\section{Mathematical Model}

The problem considered [29] involves the one-dimensional flow in a semi-infinite long porous medium with TPG for the case of a constant flow rate at the inner boundary; the porous medium is homogeneous, isotropic, and isothermal; the single-phase horizontal flow does not have any gravity effect; and the fluid and porous medium are slightly compressible.

The continuous (mass balance) equation for the onedimensional flow in the porous medium is given as follows [20, 21, 26, 29]:

$$
\frac{\partial^{2} P_{D}}{\partial x_{D}^{2}}=\frac{\partial P_{D}}{\partial t_{D}}, \quad 0 \leq x_{D} \leq \delta\left(t_{D}\right),
$$

where $P_{D}$ is the dimensionless formation pressure, $x_{D}$ is the dimensionless distance; $t_{D}$ is the dimensionless time, and $\delta$ is the dimensionless distance of the moving boundary.

The initial conditions are as follows:

$$
\begin{gathered}
\left.P_{D}\right|_{t_{D}=0}=0, \\
\delta(0)=0 .
\end{gathered}
$$

The inner boundary condition with a constant production rate is as follows:

$$
\left.\frac{\partial P_{D}}{\partial x_{D}}\right|_{x_{D}=0}=-\left(1+\lambda_{D}\right),
$$

where $\lambda_{D}$ is the dimensionless TPG.

The moving boundary conditions are as follows [20, 21, 26, 29]:

$$
\begin{gathered}
\left.\frac{\partial P_{D}}{\partial x_{D}}\right|_{x_{D}=\delta\left(t_{D}\right)}=-\lambda_{D} \\
\left.P_{D}\right|_{x_{D}=\delta\left(t_{D}\right)}=0
\end{gathered}
$$


Equations (1)-(6) form the mathematical model for the one-dimensional flow in semi-infinite long porous media with TPG for the case of a constant flow rate at the inner boundary; from (1), (5), and (6), the velocity of the moving boundary can be deduced as follows [29]:

$$
\frac{\partial \delta}{\partial t_{D}}=\left.\frac{1}{\lambda_{D}} \cdot \frac{\partial^{2} P_{D}}{\partial x_{D}^{2}}\right|_{x_{D}=\delta\left(t_{D}\right)}
$$

\section{Numerical Solution of the Problem}

It is well known that numerical solution needs the space discretization for the flow region; however, due to the existence of the moving boundary in the model, the onedimensional flow region is not fixed but expands outside continuously with the time increasing according to (7). In order to overcome the difficulty in the space discretization for the transient flow region with the moving boundary, a spatial coordinate transformation method is introduced [3335]. Then the mathematical model with the moving boundary conditions can be transformed into a mathematical model with fixed boundary conditions. The formula of the spatial coordinate transformation is as follows:

$$
y_{D}\left(x_{D}, t_{D}\right)=\frac{x_{D}}{\delta\left(t_{D}\right)}, \quad 0 \leq x_{D} \leq \delta\left(t_{D}\right)
$$

By the spatial coordinate transformation, the following two end points in the one-dimensional spatial coordinate of $x_{D}$ can be, respectively, transformed as

$$
\begin{gathered}
y_{D}\left(0, t_{D}\right)=\frac{0}{\delta\left(t_{D}\right)}=0, \\
y_{D}\left(\delta\left(t_{D}\right), t_{D}\right)=\frac{\delta\left(t_{D}\right)}{\delta\left(t_{D}\right)}=1 .
\end{gathered}
$$

By (8), the dimensionless pressure $P_{D}\left(x_{D}, t_{D}\right)$ at the flow interval of the moving boundary model $\left[0, \delta\left(t_{D}\right)\right]$ in the one-dimensional spatial coordinate of $x_{D}$ can be equivalently transformed to the function $\widetilde{P}_{D}\left(y_{D}, t_{D}\right)$ at the fixed interval $[0,1]$ in the one-dimensional spatial coordinate of $y_{D}$, and then the differential variables in the mathematical model can be transformed, respectively, as follows:

$$
\begin{gathered}
\frac{\partial P_{D}}{\partial x_{D}}=\frac{\partial \widetilde{P}_{D}}{\partial y_{D}} \cdot \frac{1}{\delta} \\
\frac{\partial^{2} P_{D}}{\partial x_{D}^{2}}=\frac{\partial^{2} \widetilde{P}_{D}}{\partial y_{D}^{2}} \cdot \frac{1}{\delta^{2}}, \\
\frac{\partial P_{D}}{\partial t_{D}}=\frac{\partial \widetilde{P}_{D}}{\partial t_{D}}+\frac{\partial \widetilde{P}_{D}}{\partial y_{D}} \cdot\left(-\frac{x_{D}}{\delta^{2}}\right) \cdot \frac{\partial \delta}{\partial t_{D}} \\
=\frac{\partial \widetilde{P}_{D}}{\partial t_{D}}-\frac{\partial \widetilde{P}_{D}}{\partial y_{D}} \cdot \frac{\partial \delta}{\partial t_{D}} \cdot \frac{y_{D}}{\delta} .
\end{gathered}
$$

Substituting (10)-(12) into (1) yields

$$
\frac{\partial^{2} \widetilde{P}_{D}}{\partial y_{D}^{2}} \cdot \frac{1}{\delta^{2}}=\frac{\partial \widetilde{P}_{D}}{\partial t_{D}}-\frac{\partial \widetilde{P}_{D}}{\partial y_{D}} \cdot \frac{\partial \delta}{\partial t_{D}} \cdot \frac{y_{D}}{\delta}, \quad 0 \leq y_{D} \leq 1 .
$$

Substituting (10)-(12) into (4)-(7), respectively, yields

$$
\begin{gathered}
\left.\frac{\partial \widetilde{P}_{D}}{\partial y_{D}} \cdot \frac{1}{\delta}\right|_{y_{D}=0}=-\left(1+\lambda_{D}\right), \\
\left.\frac{\partial \widetilde{P}_{D}}{\partial y_{D}} \cdot \frac{1}{\delta}\right|_{y_{D}=1}=-\lambda_{D}, \\
\left.\widetilde{P}_{D}\right|_{y_{D}=1}=0, \\
\frac{\partial \delta}{\partial t_{D}}=\left.\frac{1}{\lambda_{D}} \cdot \frac{\partial^{2} \widetilde{P}_{D}}{\partial y_{D}^{2}}\right|_{y_{D}=1} \cdot \frac{1}{\delta^{2}} .
\end{gathered}
$$

Substituting (17) into (13) yields

$$
\frac{\partial^{2} \widetilde{P}_{D}}{\partial y_{D}^{2}} \cdot \frac{1}{\delta^{2}}=\frac{\partial \widetilde{P}_{D}}{\partial t_{D}}-\left.\frac{\partial \widetilde{P}_{D}}{\partial y_{D}} \cdot \frac{1}{\lambda_{D}} \cdot \frac{\partial^{2} \widetilde{P}_{D}}{\partial y_{D}^{2}}\right|_{y_{D}=1} \cdot \frac{y_{D}}{\delta^{3}}
$$

that is,

$$
\frac{\partial^{2} \widetilde{P}_{D}}{\partial y_{D}^{2}} \cdot \delta=\frac{\partial \widetilde{P}_{D}}{\partial t_{D}} \cdot \delta^{3}-\left.\frac{\partial \widetilde{P}_{D}}{\partial y_{D}} \cdot \frac{y_{D}}{\lambda_{D}} \cdot \frac{\partial^{2} \widetilde{P}_{D}}{\partial y_{D}^{2}}\right|_{y_{D}=1} .
$$

The equivalent form of (14) is as follows:

$$
\delta=-\left.\frac{\partial \widetilde{P}_{D}}{\partial y_{D}}\right|_{y_{D}=0} \cdot \frac{1}{1+\lambda_{D}}
$$

Substituting (20) into (19) to cancel the variable $\delta$ yields

$$
\begin{gathered}
\left.\frac{\partial^{2} \widetilde{P}_{D}}{\partial y_{D}^{2}} \cdot \frac{\partial \widetilde{P}_{D}}{\partial y_{D}}\right|_{y_{D}=0} \\
-\frac{1}{\left(1+\lambda_{D}\right)^{2}}\left(\left.\frac{\partial \widetilde{P}_{D}}{\partial y_{D}}\right|_{y_{D}=0}\right)^{3} \cdot \frac{\partial \widetilde{P}_{D}}{\partial t_{D}} \\
-\left.\frac{1+\lambda_{D}}{\lambda_{D}} \cdot y_{D} \cdot \frac{\partial \widetilde{P}_{D}}{\partial y_{D}} \cdot \frac{\partial^{2} \widetilde{P}_{D}}{\partial y_{D}^{2}}\right|_{y_{D}=1}=0 .
\end{gathered}
$$

From (8), (2) can be transformed as

$$
\left.\widetilde{P}_{D}\left(y_{D}, t_{D}\right)\right|_{t_{D}=0}=0 \text {. }
$$

From (14) and (15), the following equation can be deduced:

$$
\left.\frac{\partial \widetilde{P}_{D}}{\partial y_{D}}\right|_{y_{D}=0}=\left.\frac{1+\lambda_{D}}{\lambda_{D}} \frac{\partial \widetilde{P}_{D}}{\partial y_{D}}\right|_{y_{D}=1}
$$

Equations (21)-(23) and (16) form a closed system of partial differential equations with the fixed boundary conditions with respect to $\widetilde{P}_{D}\left(y_{D}, t_{D}\right)$, which is equivalent to the mathematical model for the moving boundary problem of the one-dimensional flow in semi-infinite long porous media with TPG for the case of a constant flow rate at the inner boundary. From (21), it can be seen that the partial differential equations have strong nonlinearity, which indirectly reflects 
the strong nonlinearity of the original moving boundary model. Owing to the strong nonlinearity of the transformed model, a stable, fully implicit finite difference method [36] is adopted to numerically solve the relevant nonlinear partial differential equations.

The one-dimensional unit interval is discretized as $N$ spatial grid subintervals with the same length, and then the length of every subinterval $\Delta y_{D}$ is equal to $1 / N$; the distance at the $i$ th spatial grid $y_{D i}$ is $i \cdot \Delta y_{D}$; the time step is assumed to be $\Delta t_{D}$; replace the first derivative by first-order forward difference and the second derivative by second-order central difference, and finally the fully implicit difference equations [36] corresponding to (21) can be expressed as follows:

$$
\begin{aligned}
& \frac{\widetilde{P}_{D_{i+1}}^{j+1}-2 \widetilde{P}_{D_{i}}^{j+1}+\widetilde{P}_{D_{i-1}}^{j+1}}{\left(\Delta y_{D}\right)^{2}} \cdot \frac{\widetilde{P}_{D_{1}}^{j+1}-\widetilde{P}_{D_{0}}^{j+1}}{\Delta y_{D}} \\
& -\frac{1}{\left(1+\lambda_{D}\right)^{2}}\left(\frac{\widetilde{P}_{D_{1}}^{j+1}-\widetilde{P}_{D_{0}}^{j+1}}{\Delta y_{D}}\right)^{3} \cdot \frac{\widetilde{P}_{D_{i}}^{j+1}-\widetilde{P}_{D_{i}}^{j}}{\Delta t_{D}} \\
& -\frac{1+\lambda_{D}}{\lambda_{D}} \cdot i \cdot \Delta y_{D} \cdot \frac{\widetilde{P}_{D_{i+1}}^{j+1}-\widetilde{P}_{D_{i}}^{j+1}}{\Delta y_{D}} \cdot \frac{\widetilde{P}_{D_{N-2}}^{j+1}-2 \widetilde{P}_{D_{N-1}}^{j+1}}{\left(\Delta y_{D}\right)^{2}}=0 \\
& \quad(i=1,2, \ldots, N-2),
\end{aligned}
$$

where $j$ denotes the index of time step.

The difference equation of (16) is as follows:

$$
\widetilde{P}_{D_{N}}^{j+1}=0 .
$$

Then, by (25), the difference equation corresponding to the $(N-1)$ th spatial grid can be expressed as follows:

$$
\begin{aligned}
& \frac{-2 \widetilde{P}_{D_{N-1}}^{j+1}+\widetilde{P}_{D_{N-2}}^{j+1}}{\left(\Delta y_{D}\right)^{2}} \cdot \frac{\widetilde{P}_{D_{1}}^{j+1}-\widetilde{P}_{D_{0}}^{j+1}}{\Delta y_{D}} \\
& \quad-\frac{1}{\left(1+\lambda_{D}\right)^{2}}\left(\frac{\widetilde{P}_{D_{1}}^{j+1}-\widetilde{P}_{D_{0}}^{j+1}}{\Delta y_{D}}\right)^{3} \cdot \frac{\widetilde{P}_{D_{N-1}}^{j+1}-\widetilde{P}_{D_{N-1}}^{j}}{\Delta t_{D}} \\
& \quad+\frac{1+\lambda_{D}}{\lambda_{D}} \cdot(N-1) \cdot \Delta y_{D} \cdot \frac{\widetilde{P}_{D_{N-1}}^{j+1}}{\Delta y_{D}} \cdot \frac{\widetilde{P}_{D_{N-2}}^{j+1}-2 \widetilde{P}_{D_{N-1}}^{j+1}}{\left(\Delta y_{D}\right)^{2}}=0 .
\end{aligned}
$$

The difference equation of (23) is as follows:

$$
\frac{\widetilde{P}_{D_{1}}^{j+1}-\widetilde{P}_{D_{0}}^{j+1}}{\Delta y_{D}}=-\frac{1+\lambda_{D}}{\lambda_{D}} \frac{\widetilde{P}_{D_{N-1}}^{j+1}}{\Delta y_{D}} .
$$

From (22), the initial values of the unknown dimensionless formation pressure are as follows:

$$
\widetilde{P}_{D_{i}}^{0}=0, \quad i=0,1,2, \ldots, N-1 .
$$

From (24), (26), and (27), it can be seen that there are $N$ difference equations at the $(j+1)$ th time step, which contain
$N$ unknown variables $\widetilde{P}_{D_{i}}^{j+1}(i=0,1,2, \ldots, N-1)$; due to the strong nonlinearity of these difference equations, the NewtonRaphson iteration method [36] is adopted to obtain their numerical solutions; when $\widetilde{P}_{D_{i}}^{j+1}(i=0,1,2, \ldots, N-1)$ are numerically solved for, let $j$ be equal to $j+1$, and in the same manner the numerical solutions of $N$ difference equations with respect to $N$ unknown variables $\widetilde{P}_{D_{i}}^{j+2}(i=0,1,2, \ldots, N-$ $1)$ at the $(j+2)$ th time step can also be numerically solved for; the rest can be deduced by analogy; then the numerical solutions of the nonlinear partial differential equations with respect to $\widetilde{P}_{D}\left(y_{D}, t_{D}\right)$ can be obtained.

The difference equation of $(8)$ is

$$
x_{D_{i}}^{j+1}=y_{D_{i}} \cdot \delta^{j+1} .
$$

The difference equation of (20) is

$$
\delta^{j+1}=-\frac{\widetilde{P}_{D_{1}}^{j+1}-\widetilde{P}_{D_{0}}^{j+1}}{\Delta y_{D}} \cdot \frac{1}{1+\lambda_{D}} .
$$

Substituting (30) into (29) yields

$$
x_{D_{i}}^{j+1}=-i \cdot \frac{\widetilde{P}_{D_{1}}^{j+1}-\widetilde{P}_{D_{0}}^{j+1}}{1+\lambda_{D}} .
$$

From (31), in the process of numerical solutions at every time step, the one-dimensional spatial coordinate of $y_{D}$ can be transformed as the one of $x_{D}$, and then the numerical solutions of $\widetilde{P}_{D}\left(y_{D}, t_{D}\right)$ can be transformed as the numerical solutions of $P_{D}\left(x_{D}, t_{D}\right)$. From (31), it can also be seen that it is just the spatial coordinate transformation, that is (8), that lets the time-dependent space discretization in the spatial coordinate of $x_{D}$ be transformed as a time-independent space discretization in the spatial coordinate of $y_{D}$, which makes the numerical solutions by the finite difference method more applicable and simple.

\section{Verification of Numerical Solutions}

4.1. Exact Analytical Solutions. The exact analytical solution of the mathematical model for the one-dimensional flow in semi-infinite long porous media with TPG for the case of a constant flow rate at the inner boundary is as follows [29]:

$$
\begin{aligned}
& P_{D}\left(x_{D}, t_{D}\right) \\
& =2\left(1+\lambda_{D}\right) \\
& \quad \times\left[\theta\left(\frac{t_{D}{ }^{1 / 2} e^{-x_{D}^{2} / 4 / t_{D}}+x_{D} / 2 \pi^{1 / 2} \operatorname{erf}\left(x_{D} / 2 / \sqrt{t_{D}}\right)}{e^{-\theta^{2}}+\pi^{1 / 2} \theta \operatorname{erf}(\theta)}\right)-\frac{x_{D}}{2}\right], \\
& x_{D} \in[0, \delta],
\end{aligned}
$$

where $\theta$ can be determined by the following equation with respect to the TPG [29]:

$$
\frac{e^{-\theta^{2}}}{e^{-\theta^{2}}+\pi^{1 / 2} \theta \operatorname{erf}(\theta)}=\frac{\lambda_{D}}{1+\lambda_{D}} .
$$


TABLE 1: Calculation of dimensionless TPG $\lambda_{D}$ through experimental data [2] of eight samples.

\begin{tabular}{lcccccc}
\hline Sample & $\begin{array}{c}\lambda \\
(\mathrm{psi} / \mathrm{cm})\end{array}$ & $\begin{array}{c}k / \mu \\
(\mathrm{md} / \mathrm{cp})\end{array}$ & $\begin{array}{c}\text { Minimum flow } \\
\text { rate } q_{1}\left(\mathrm{~cm}^{3} / \mathrm{min}\right)\end{array}$ & $\begin{array}{c}\text { Maximum flow } \\
\text { rate } q_{2}\left(\mathrm{~cm}^{3} / \mathrm{min}\right)\end{array}$ & $\begin{array}{c}\text { Cross-sectional } \\
\text { area } A\left(\mathrm{~cm}^{2}\right)\end{array}$ & $\begin{array}{c}\text { Average flow velocity } \\
\nu_{w}=\left(q_{1}+q_{2}\right) / A / 2 \\
\left(\mathrm{~cm} / \mathrm{min}^{2}\right.\end{array}$ \\
\hline Brown sandstone \#1 & 0.00846 & 6520 & 8.83 & 30 & 20.2 & 0.961139 \\
$\lambda_{D}=\lambda k / \mu / \nu_{w}$
\end{tabular}

It has been proven [29] that, for a given value of $\lambda_{D}$, there is one and only one positive root of (33).

The distance of the moving boundary can be expressed as follows [29]:

$$
\delta=2 \cdot \theta \cdot \sqrt{t_{D}}
$$

When $\lambda_{D}=0$, the exact analytical solution of the mathematical model for the one-dimensional Darcy's flow in semiinfinite long porous media for the case of a constant flow rate at the inner boundary is as follows [29]:

$$
\begin{aligned}
P_{D}\left(x_{D}, t_{D}\right)= & -x_{D}+2 \sqrt{\frac{t_{D}}{\pi}} \exp \left(\frac{-x_{D}^{2}}{4 t_{D}}\right) \\
& +x_{D} \operatorname{erf}\left(\frac{x_{D}}{2 \sqrt{t_{D}}}\right), \quad x_{D} \in[0, \infty] .
\end{aligned}
$$

4.2. Assignment of the Values of Dimensionless TPG $\lambda_{D}$. The formula for the dimensionless TPG $\lambda_{D}$ is as follows [29]:

$$
\lambda_{D}=\frac{k}{\mu} \frac{\lambda}{\nu_{w}},
$$

where $k$ is the permeability of porous media, $\lambda$ is the TPG, $\mu$ is the fluid viscosity, and $\nu_{w}$ is the average seepage velocity.

Here, selected Prada and Civan's actual experimental data of eight samples [2] for measuring the one-dimensional flow in three types of porous media (brown sandstone, sandpack, and shaly sandstone) with TPG is used to calculate the values of $\lambda_{D}$; the experimental data and specific calculation process are shown in Table 1 .

From Table 1, it can be concluded that the range of the values of dimensionless TPG $\lambda_{D}$ is between 0.242 and 0.852 for the eight samples of three types of porous media. Without loss of generality, the values of dimensionless TPG $\lambda_{D}$ are set $0.242,0.553$, and 0.852 for the following numerical tests, respectively.

4.3. Numerical Tests. By the numerical method presented in the paper, the mathematical model for the one-dimensional flow in semi-infinite long porous media with TPG for the case of a constant flow rate at the inner boundary is numerically

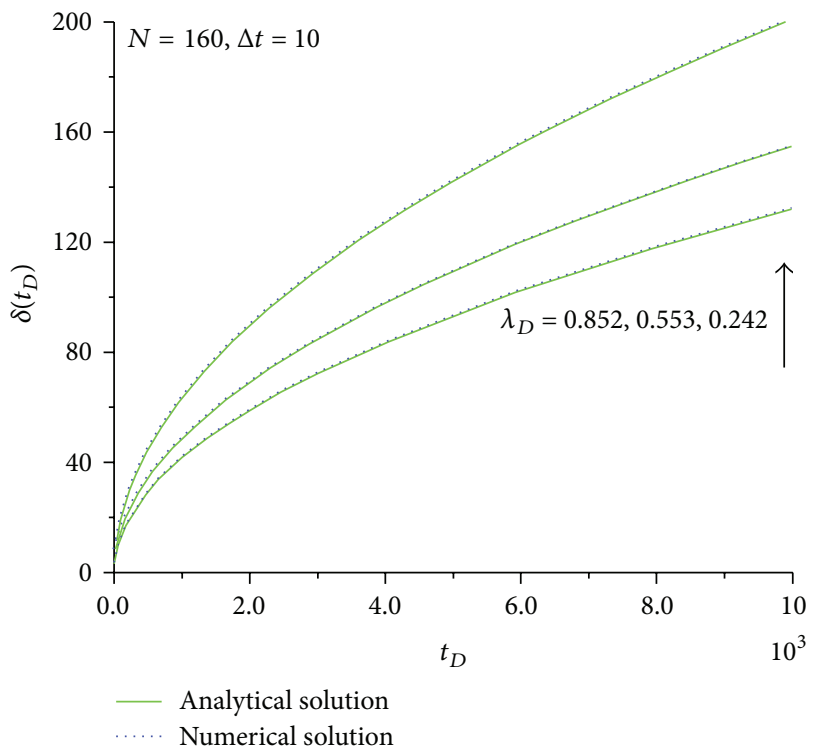

FIgURE 1: Comparison of transient distance of moving boundary under different values of dimensionless TPG.

solved, where $N=160, \Delta t_{D}=10$, and $\lambda_{D}=0.242,0.553$, and 0.852 , respectively. By (33), the values of $\theta$ corresponding to the three values of $\lambda_{D}$ can be computed by NewtonRaphson iteration method [36] as 1.005, 0.7732, and 0.6599 for the exact analytical solutions. In order to make clear the difference of model solutions between Darcy's flow, when $\lambda_{D}=0$, and the fluid flow in porous media with the TPG, exact analytical solutions for the Darcy's flow, that is, (35), are also added for the purpose of comparison analysis.

Under different values of $\lambda_{D}$, the numerical results are graphically compared with those from the exact analytical solution, that is, (32)-(34). Figures 1-3 show the comparison curves between the numerical solutions and the exact analytical solutions with respect to the transient distance of the moving boundary, the transient production pressure of wellbore, and the formation pressure distribution when $t_{D}=$ 5,000 , respectively.

From Figures 1-3, it can be seen that the numerical solutions are in good agreement with the exact analytical 


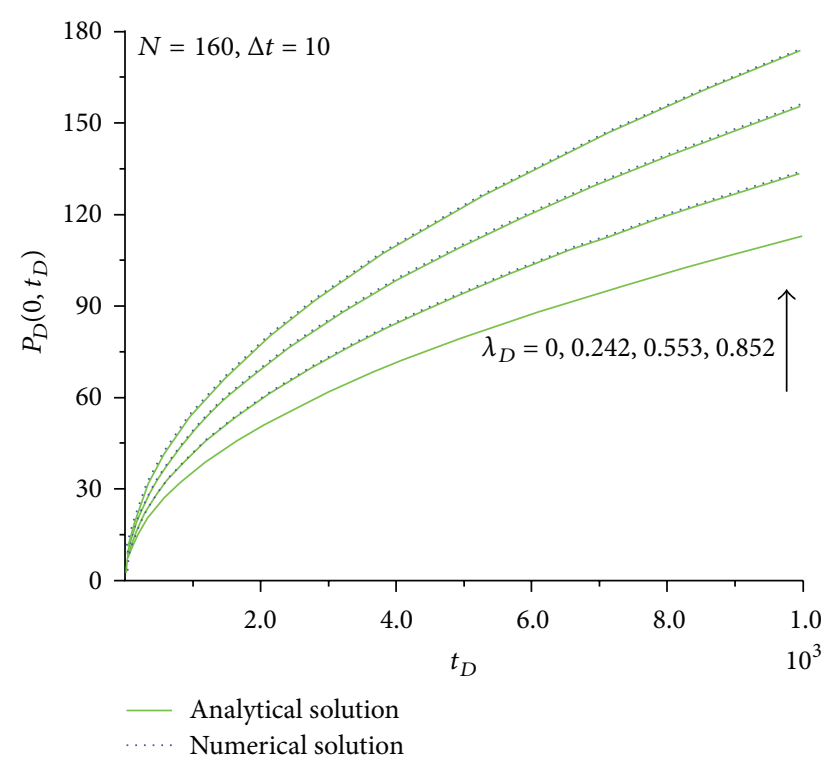

FIGURE 2: Comparison of transient production pressure of wellbore under different values of dimensionless TPG.

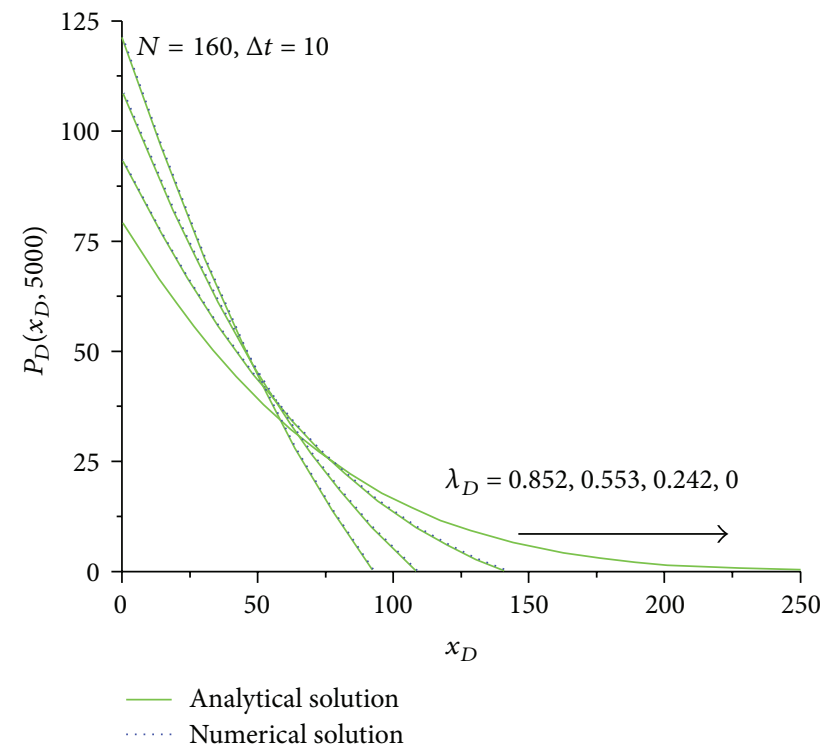

FIGURE 3: Comparison of formation pressure distribution when $t_{D}$ $=5,000$ under different values of dimensionless TPG.

solutions for the moving boundary problem. Furthermore, through lots of numerical tests, it is found that the accuracy of the numerical solutions can be further improved by increasing the value of $N$. It demonstrates the correctness and validity of the presented numerical method here for solving the moving boundary problem of the one-dimensional flow in semi-infinite long porous media with TPG.

Besides, from Figures 2 and 3, it can also be concluded that the TPG has a big influence on the model solutions; the larger the value of the dimensionless TPG, the larger the difference of model solutions between Darcy's flow and fluid flow in porous media with TPG; in Figure 3, the formation pressure distribution curves corresponding to non-zero dimensionless TPG indicate an instructive characteristics of model solutions: they have compact supports [1], which are different from that of Darcy's flow. Therefore, it is very necessary to take into account the effect of TPG for the fluid flow in porous media with TPG, for mathematical modeling and engineering applications, especially for the case of a large dimensionless TPG that is, (36).

\section{Conclusions}

The utility of the presented numerical method can be attributed to its simple approach of spatial coordinate transformation in numerically solving the moving boundary problem effectively by the stable, fully implicit finite difference method. The accuracy of the numerical solutions is verified by graphically comparing with the published exact analytical solutions. Besides, the accuracy can be further improved by increasing the value of $N$, that is, decreasing the length of the spatial grid subintervals. The numerical results also show the big difference of model solutions between Darcy's flow and the fluid flow in porous media with TPG, especially for the case of a large dimensionless TPG. In comparison with previous numerical methods for solving such moving boundary problems, the verified numerical method presented here is more reliable and convenient to implement by the finite difference method in developing well testing software and reservoir simulator. The numerical method will be applicable to the moving boundary problems of multi-dimensional flow in porous media with TPG, which will be our future research topic. The presented research supports theoretical foundations for technologies of well testing and numerical simulation in developing low-permeable reservoirs and heavy oil reservoirs.

\section{Conflict of Interests}

Jun Yao, Wenchao Liu, and Zhangxin Chen declare that there is no conflict of interests regarding the publication of this paper.

\section{Acknowledgments}

The authors would like to acknowledge the funding by the project (Grant no. 11102237) sponsored by the Natural Science Foundation of China (NSFC) and the Program for Changjiang Scholars and Innovative Research Team in University (Grant no. IRT1294). In particular, Wenchao Liu also would like to express his great gratitude to the China Scholarship Council (CSC) for the generous financial support of the research.

\section{References}

[1] P. J. M. Monteiro, C. H. Rycroft, and G. I. Barenblatt, "A mathematical model of fluid and gas flow in nanoporous media," Proceedings of the National Academy of Sciences of the United States of America, vol. 109, no. 50, pp. 20309-20313, 2012. 
[2] A. Prada and F. Civan, "Modification of Darcy's law for the threshold pressure gradient," Journal of Petroleum Science and Engineering, vol. 22, no. 4, pp. 237-240, 1999.

[3] F. Civan, Porous Media Transport Phenomena, John Wiley and Sons, Hoboken, NJ, USA, 2011.

[4] S. Wang, Y. Huang, and F. Civan, "Experimental and theoretical investigation of the Zaoyuan field heavy oil flow through porous media," Journal of Petroleum Science and Engineering, vol. 50, no. 2, pp. 83-101, 2006.

[5] F. Hao, L. S. Cheng, O. Hassan, J. Hou, C. Z. Liu, and J. D. Feng, "Threshold pressure gradient in ultra-low permeability reservoirs," Petroleum Science and Technology, vol. 26, no. 9, pp. 1024-1035, 2008.

[6] X. Wei, L. Qun, G. Shusheng, H. Zhiming, and X. Hui, "Pseudo threshold pressure gradient to flow for low permeability reservoirs," Petroleum Exploration and Development, vol. 36, no. 2, pp. 232-236, 2009.

[7] W. Jing, L. Huiqing, P. Zhanxi, L. Renjing, and L. Ming, “The investigation of threshold pressure gradient of foam flooding in porous media," Petroleum Science and Technology, vol. 29, no. 23, pp. 2460-2470, 2011.

[8] Y. D. Yao and J. L. Ge, "Characteristics of non-Darcy flow in low-permeability reservoirs," Petroleum Science, vol. 8, no. 1, pp. $55-62,2011$

[9] F.-Q. Song, J.-D. Wang, and H.-L. Liu, "Static threshold pressure gradient characteristics of liquid influenced by boundary wettability," Chinese Physics Letters, vol. 27, no. 2, Article ID 024704, 2010.

[10] X.-W. Wang, Z.-M. Yang, Y.-D. Qi, and Y.-Z. Huang, "Effect of absorption boundary layer on nonlinear flow in low permeability porous media," Journal of Central South University of Technology, vol. 18, no. 4, pp. 1299-1303, 2011.

[11] B. Zeng, L. Cheng, and C. Li, "Low velocity non-linear flow in ultra-low permeability reservoir," Journal of Petroleum Science and Engineering, vol. 80, no. 1, pp. 1-6, 2012.

[12] M. Yun, B. Yu, and J. Cai, "A fractal model for the starting pressure gradient for Bingham fluids in porous media," International Journal of Heat and Mass Transfer, vol. 51, no. 5-6, pp. 1402-1408, 2008.

[13] Y. Li and B. Yu, "Study of the starting pressure gradient in branching network," Science China Technological Sciences, vol. 53, no. 9, pp. 2397-2403, 2010.

[14] J. C. Cai, B. M. Yu, M. Q. Zou, and M. Mei, "Fractal analysis of invasion depth of extraneous fluids in porous media," Chemical Engineering Science, vol. 65, no. 18, pp. 5178-5186, 2010.

[15] J. Cai, B. Yu, M. Zou, and L. Luo, "Fractal characterization of spontaneous co-current imbibition in porous media," Energy and Fuels, vol. 24, no. 3, pp. 1860-1867, 2010.

[16] W. Zhu, H. Song, X. Huang, X. Liu, D. He, and Q. Ran, "Pressure characteristics and effective deployment in a water-bearing tight gas reservoir with low-velocity non-darcy flow," Energy and Fuels, vol. 25, no. 3, pp. 1111-1117, 2011.

[17] Y. Zhu, J.-Z. Xie, W.-H. Yang, and L.-H. Hou, "Method for improving history matching precision of reservoir numerical simulation," Petroleum Exploration and Development, vol. 35, no. 2, pp. 225-229, 2008.

[18] D.-Y. Yin and H. Pu, "Numerical simulation study on surfactant flooding for low permeability oilfield in the condition of threshold pressure," Journal of Hydrodynamics, vol. 20, no. 4, pp. 492-498, 2008.
[19] H. Pascal, "Nonsteady flow through porous media in the presence of a threshold gradient," Acta Mechanica, vol. 39, no. 3-4, pp. 207-224, 1981.

[20] Y. S. Wu, K. Pruess, and P. A. Witherspoon, "Flow and displacement of Bingham non-Newtonian fluids in porous media," SPE Reservoir Engineering, vol. 7, no. 3, pp. 369-376, 1992.

[21] S. Fuquan, L. Ciqun, and L. Fanhua, "Transient pressure of percolation through one dimension porous media with threshold pressure gradient," Applied Mathematics and Mechanics, vol. 20, no. 1, pp. 27-35, 1999.

[22] J. Nedoma, "Numerical solution of a Stefan-like problem in Bingham rheology," Mathematics and Computers in Simulation, vol. 61, no. 3-6, pp. 271-281, 2003.

[23] M. Chen, W. Rossen, and Y. C. Yortsos, "The flow and displacement in porous media of fluids with yield stress," Chemical Engineering Science, vol. 60, no. 15, pp. 4183-4202, 2005.

[24] G.-Q. Feng, Q.-G. Liu, G.-Z. Shi, and Z.-H. Lin, "An unsteady seepage flow model considering kickoff pressure gradient for low-permeability gas reservoirs," Petroleum Exploration and Development, vol. 35, no. 4, pp. 457-461, 2008.

[25] I. Daprà and G. Scarpi, "Unsteady simple shear flow in a viscoplastic fluid: comparison between analytical and numerical solutions," Rheologica Acta, vol. 49, no. 1, pp. 15-22, 2010.

[26] K.-H. Xie, K. Wang, Y.-L. Wang, and C.-X. Li, "Analytical solution for one-dimensional consolidation of clayey soils with a threshold gradient," Computers and Geotechnics, vol. 37, no. 4, pp. 487-493, 2010.

[27] W. J. Luo and X. D. Wang, "Effect of a moving boundary on the fluid transient flow in low permeability reservoirs," Journal of Hydrodynamics B, vol. 24, no. 3, pp. 391-398, 2012.

[28] X. Wang, X. Hou, M. Hao, and T. Yang, "Pressure transient analysis in low-permeable media with threshold gradients," Acta Petrolei Sinica, vol. 32, no. 5, pp. 847-851, 2011.

[29] W. C. Liu, J. Yao, and Y. Y. Wang, "Exact analytical solutions of moving boundary problems of one-dimensional flow in semiinfinite long porous media with threshold pressure gradient," International Journal of Heat Mass Transfer, vol. 55, no. 21-22, pp. 6017-6022, 2012.

[30] W. C. Liu, J. Yao, Z. X. Sun et al., "Model of nonlinear seepage flow in low-permeability porous media based on the permeability gradual theory," Chinese Journal of Computational Mechanics, vol. 29, no. 6, pp. 885-892, 2012.

[31] S. L. Marshall, "Nonlinear pressure diffusion in flow of compressible liquids through porous media," Transport in Porous Media, vol. 77, no. 3, pp. 431-446, 2009.

[32] R. Wang, X. Yue, R. Zhao, P. Yan, and F. Dave, "Effect of stress sensitivity on displacement efficiency in $\mathrm{CO}_{2}$ flooding for fractured low permeability reservoirs," Petroleum Science, vol. 6, no. 3, pp. 277-283, 2009.

[33] J. Crank, Free and Moving Boundary Problems, Clarendon Press, Oxford, UK, 1984.

[34] R. S. Gupta and A. Kumar, "Treatment of multi-dimensional moving boundary problems by coordinate transformation," International Journal of Heat and Mass Transfer, vol. 28, no. 7, pp. 1355-1366, 1985.

[35] G. Senthil, G. Jayaraman, and A. D. Rao, "A variable boundary method for modelling two dimensional free surface flows with moving boundaries," Applied Mathematics and Computation, vol. 216, no. 9, pp. 2544-2558, 2010.

[36] R. L. Burden and J. D. Faires, Numerical Analysis, Brooks Cole, Florence, Ky, USA, 9th edition, 2010. 


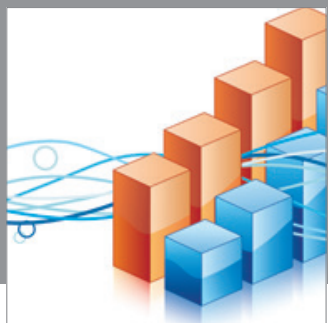

Advances in

Operations Research

mansans

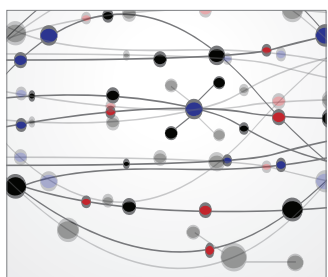

The Scientific World Journal
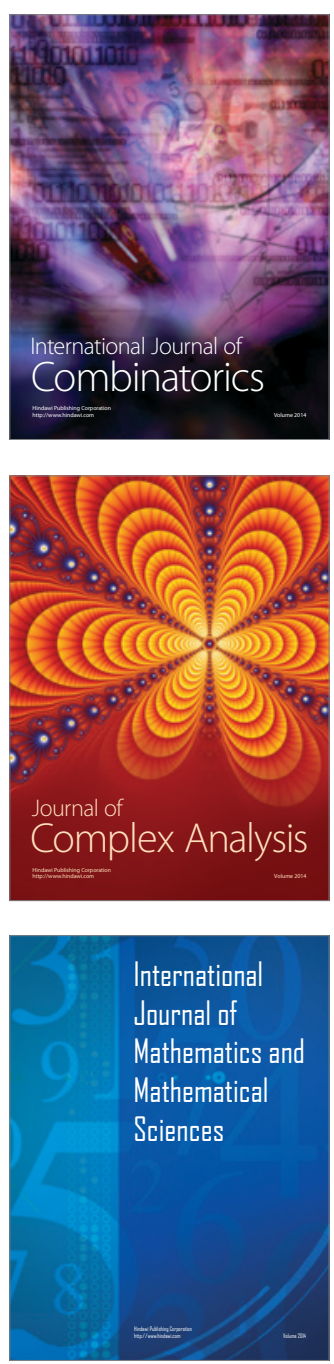
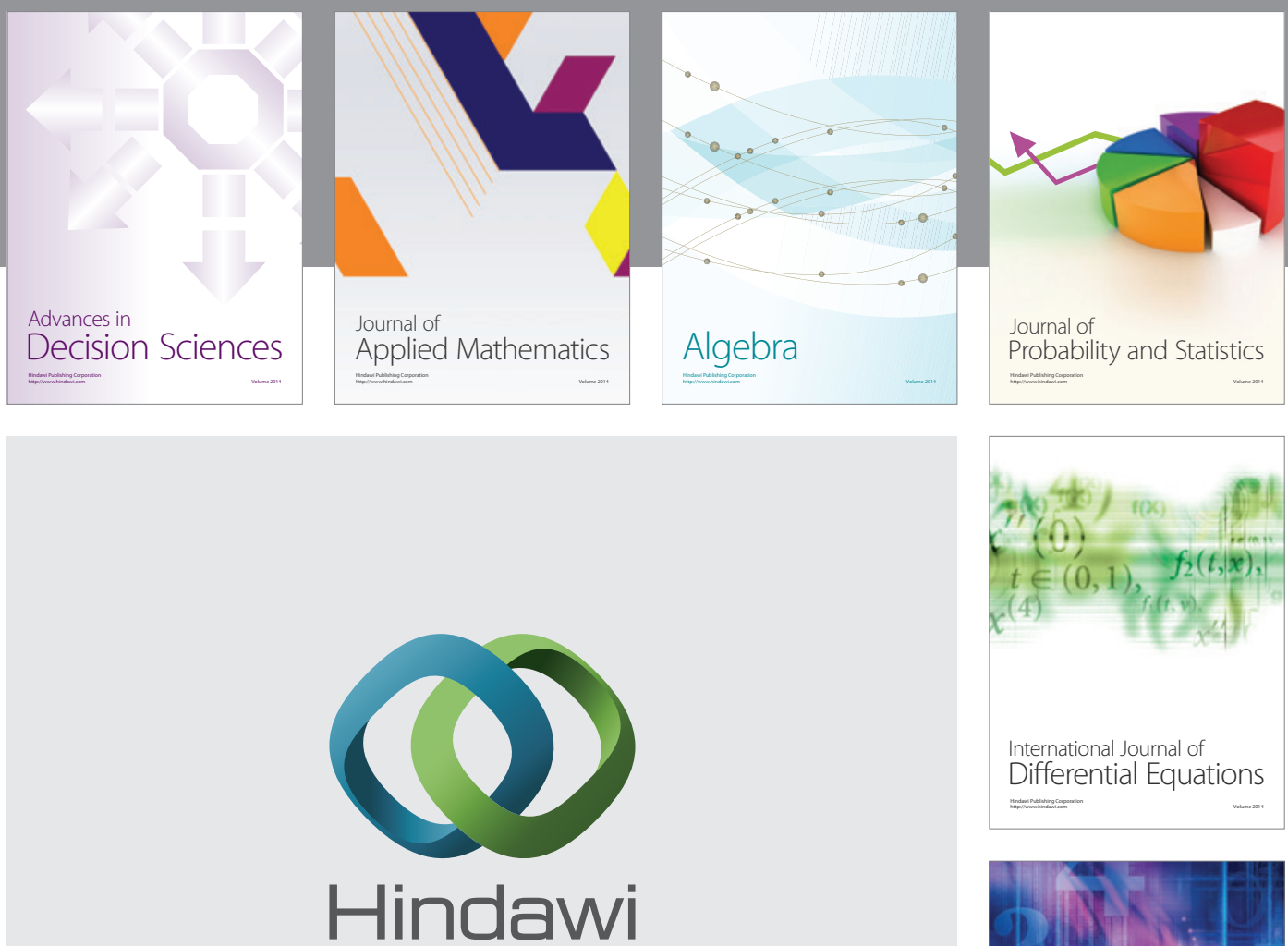

Submit your manuscripts at http://www.hindawi.com
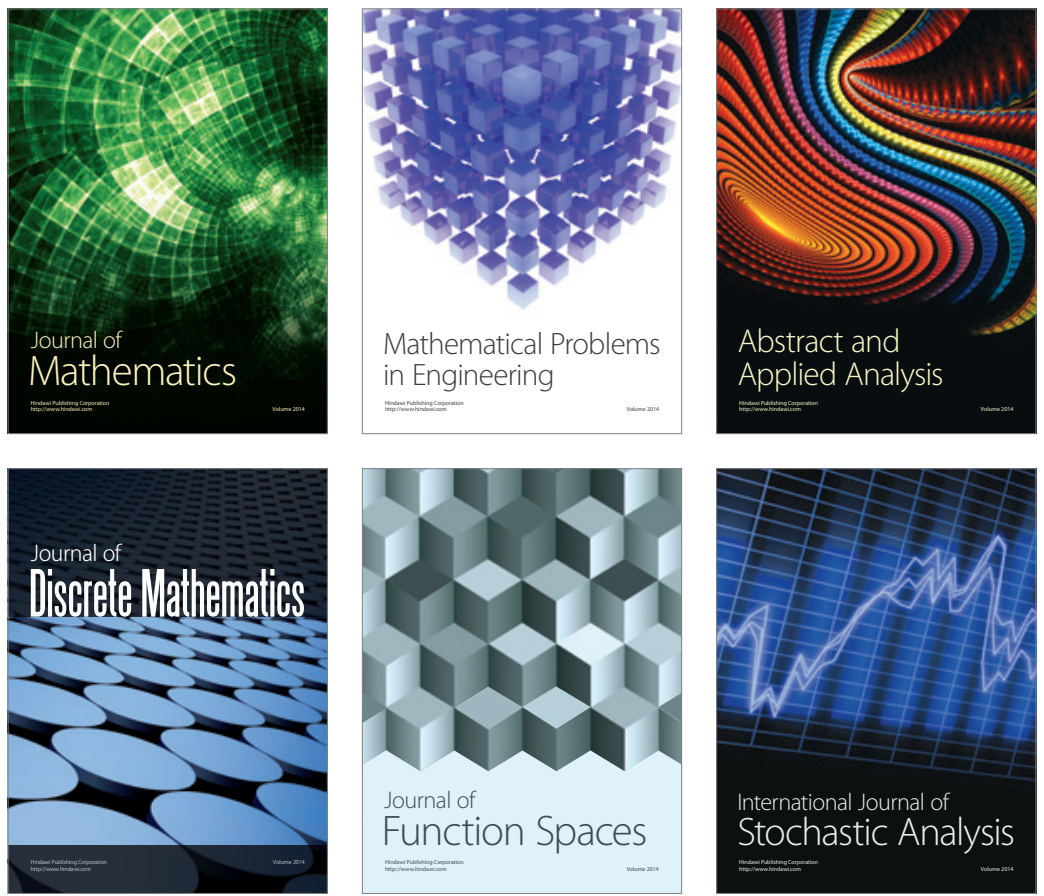

Journal of

Function Spaces

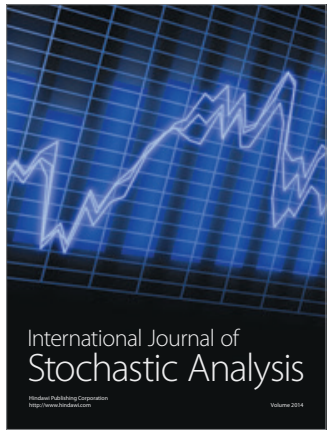

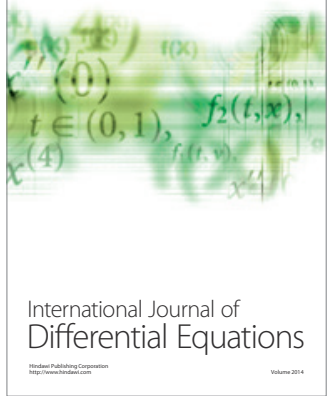
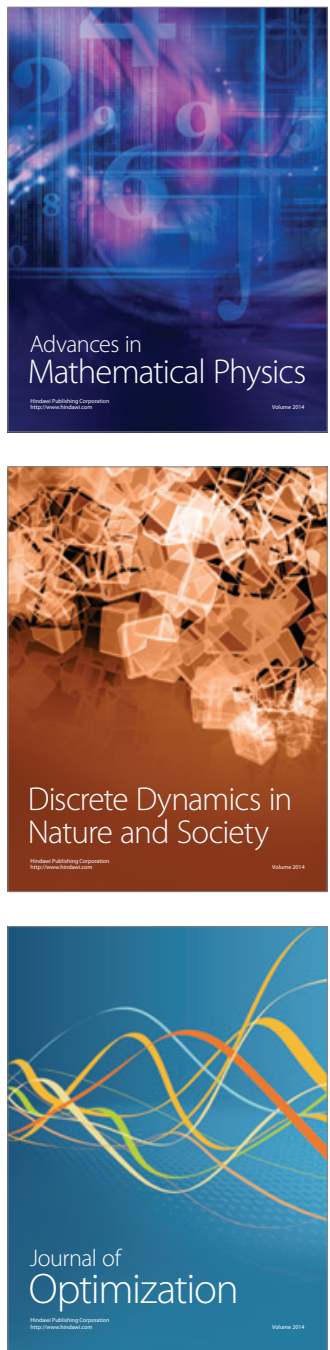